\title{
Heydemann interpolation correction for energy linearization of soft $\mathrm{X}$-ray monochromators.
}

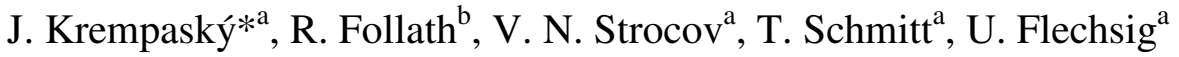 \\ ${ }^{\mathrm{a}}$ Swiss Light Source, Paul Scherrer Institut; 5232 Villigen PSI, Switzerland \\ ${ }^{\mathrm{b}}$ Helmholtz-Center Berlin, Electron Storage Ring BESSY II, Albert-Einstein-Straße 15, 12489 Berlin, Germany
}

\begin{abstract}
Material science research in the soft-X ray regime at the Swiss Light Source accommodates five beamlines where the monochromators rely on in-vacuum angular encoders for positioning mirror and gratings. Despite the factory-calibration of the quadrature signals from these rotary encoders, the energy linearization for spectroscopic data requires accurate calibration of the encoder quadrature signals. We characterize the interpolation errors and describe the Heydemann correction algorithm for the quadrature signals for improving the energy linearization on a scale comparable with the incremental encoder interpolation interval. Experimental data are shown where such errors produce sizeable effects in soft-X ray spectroscopy and for which the correction algorithm efficiently improves the short-range non-linearity.
\end{abstract}

Keywords: monochromator, energy linearization, incremental encoder, interpolation correction

\section{INTRODUCTION}

The Swiss Light Source at Paul Scherrer Institute is a 3rd generation synchrotron radiation light source with energy of $2.4 \mathrm{GeV}$ providing photon beams of high brightness for research in materials science, biology and chemistry. By the end of 2011 the number of beamlines operating in the soft X-ray regime will be eight; five of them will accommodate plane grating monochromators (PGM). The general concept of these beamlines is based on a horizontally deflecting mirror collimating the beam in the vertical direction. The collimated beam allows varying the fix-focus constant (magnification ratio $c$ ) as a free parameter without the need to move the exit slit. After 10 years of operation the beamline commissioning process became a well defined procedure. For example the PGM energy calibration consists in taking spectra for different settings such that the mirror (grating) angle $\theta(\beta)$ satisfy the grating equation

$$
m N \lambda=2 \cos \theta \sin (\beta+\theta),
$$

where $m$ is the diffraction order, $N$ is the grating line density and $\lambda$ is the required wavelength. In practical terms for every grating a set of constant offsets for the absolute angles $\theta$ and $\beta$ have to be fixed in order to calibrate energy over a wide range. However this approach is not sufficient to guarantee energy linearization on a smaller scale $(\sim 1 \mathrm{eV})$. It turns out that the main reason for this are the interpolation errors of the incremental encoders. A simple verification of the absolute angular linearity readout can be made with an autocollimator. In such measurement setup the autocollimator measures the angles of an auxiliary mirror mounted on the mirror or grating stage. The autocollimator beam is reflected from the mirror (or grating) stage. The angular deflections are recorded by scanning the stage with incremental steps much smaller than the encoder interpolation interval. In Fig.1a-b displays a comparison between the absolute angular readout from the autocollimator (a) and encoder (b) when the interpolation correction method is turned on and off. In order to linearize the angular readout (red line in Fig1.a) the motion control has to have a specific wiggling (red line Fig1b). The specific wiggling is determined by the Heydemann correction algorithm [1,2]. The eight peaks and dips over an angular extension of $0.04^{\circ}$ are related to the interpolation errors which take place within one interpolation angular span of $0.01^{\circ}$. Hence two consecutive peak-dip structures are attributed to residua $\Delta$ from the ideal linear angular readout from one interpolation interval. It should be noted that without the interpolation correction the absolute errors in the angular go beyond the mirror slope error contributions which are of the order of $\pm 0.1 \operatorname{arcsec}$.

*juraj.krempasky@psi.ch; phone ++41 56 3105131; fax ++41 56 3105313; www.psi.ch

Advances in X-Ray/EUV Optics and Components VI, edited by Christian Morawe, Ali M. Khounsary, Shunji Goto, Proc. of SPIE Vol. 8139, 81390K - @ 2011 SPIE · CCC code: 0277-786X/11/\$18

doi: $10.1117 / 12.893464$

Proc. of SPIE Vol. $813981390 \mathrm{~K}-1$ 


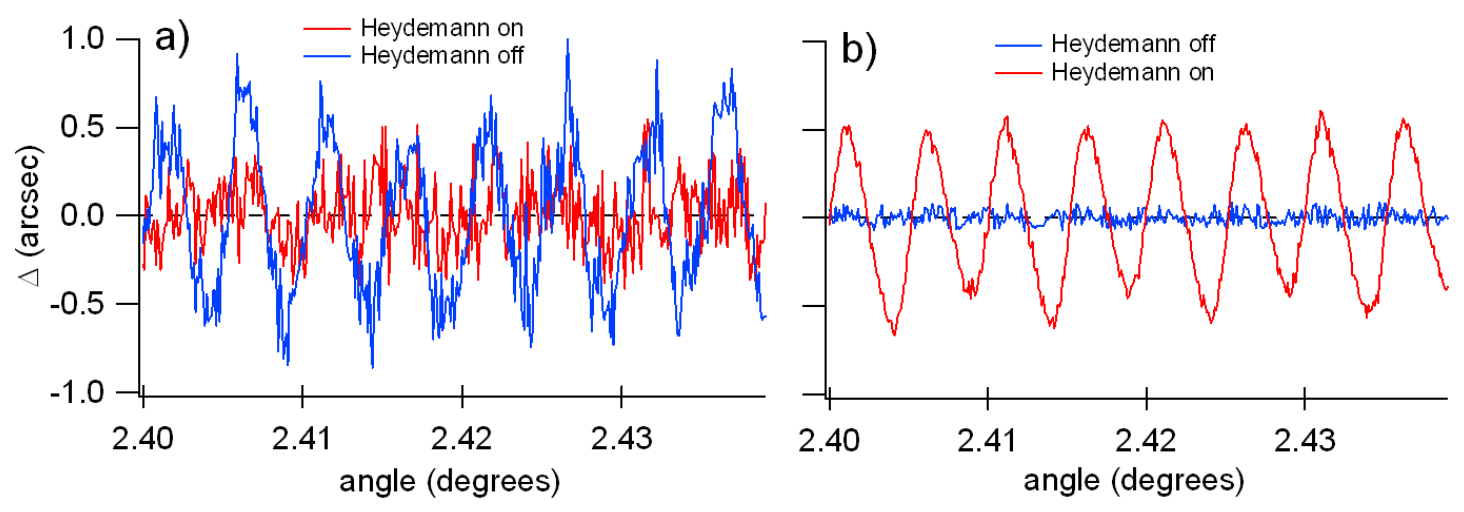

Figure 1. Single encoder head linear regression analysis from autocollimator (a) and angular incremental encoder (b) when the Heydemann correction is turned on and off ( $\Delta$ are deviations from the ideal linear angular readout).

We show that the interpolation errors do not impact on the monochromator energy resolution, but shift the energy on a scale larger than the spectroscopic resolution.

\section{RON 905 INCREMENTAL ENCODER SYSTEM}

The RON 905 system for absolute angular readouts has four independent heads mounted on a graduated disc with angular separation of $90^{\circ}$ [3]. This solution compensates errors due to excentric mount of the whole encoder system. The resulting absolute angle is an average from the four encoder heads. The system allows to readout the analog signals used for the interpolations for each head. In the ideal case the signals should have the same amplitude and should be phaseshifted by $90^{\circ}$ to give the sinus and cosinus analog outputs for the quadrature process. By plotting them as shown in Fig.2a it is possible to verify to which extent the signals deviate from the ideal conditions: the more ellipsoidal shape it has, the more severe are the interpolation errors after the quadrature process. It turns out that all encoder heads used in all of our PGMs have ellipsoidal shapes which inside the interpolation process induce sizeable errors in the angular readout. In principle the RON 905 system is capable to compensate these errors. However such a compensation is not applicable in our case due to the mechanical constrains (the system allows to move over a limited angular span with limited velocity). For this reason the interpolation errors have to be corrected after the quadrature process.

In the PGM software motion control the 4-head averaged absolute angle is acting in a "closed-loop" linear translation motion for setting the requested angle. It is clear that when all encoders are involved in the closed-loop feedback, it is difficult to disentangle the errors induced from individual heads. This is seen in Fig $2 \mathrm{~b}$ : if the closed-loop feedback is considering just one head at a time (blue line), the feedback is not influenced by other heads, the encoder shows a linear trend and the autocollimator measures periodic wiggles from interpolation errors (Fig.1a). If all four heads are averaged, the feedback loop is wiggling (Fig.2b red line) but without angular linearization (not shown). For these reasons for the correction analysis encoder quadrature signals have to be recorded for each head separately and the Heydemann correction has to be applied independently before averaging the angles. 

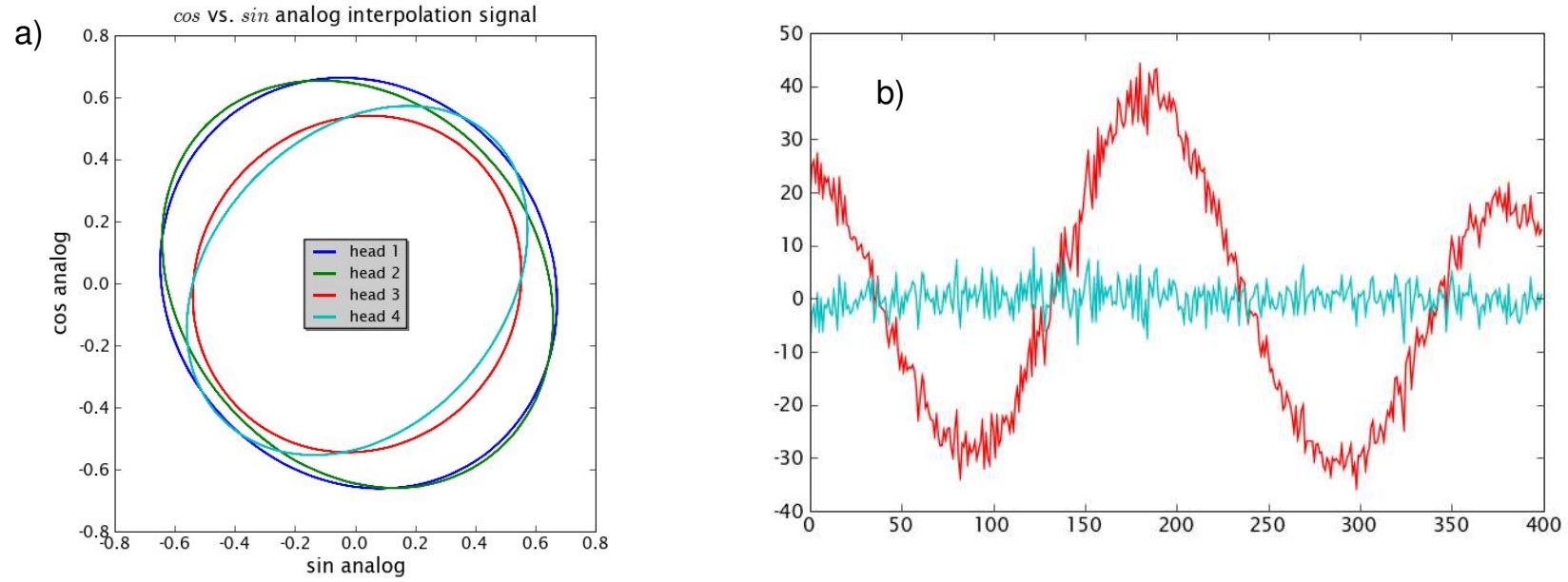

Measurement points within the $0.01^{\circ}$ interpolation span

Figure 2. (a) Analog interpolation signals plotted against each other for each encoder head. (b) Linear regression encoder counts residua from one interpolation interval: (blue line) from one encoder head; (red-line) from the same encoder head in a closed-loop feedback with all four encoders.

\section{THE HEYDEMANN CORRECTION}

For the Heydemann correction we assume that the quadrature signals have different amplitudes $A_{A}, A_{B}$, their relative phase shift is not $\pi / 2$, we consider phases $\phi_{A}, \phi_{B}$. Finally they are non-symmetric around zero (different off-sets $\left.O_{A}, O_{B}\right):$

$$
\begin{aligned}
& A=O_{A}+A_{A} \sin \left(\phi+\phi_{A}\right) \\
& B=O_{B}+A_{B} \cos \left(\phi+\phi_{B}\right),
\end{aligned}
$$

Fig.3 illustrates this procedure: $\sin (\phi)$ and $\cos (\phi)$ analog signals (raw data) are fit with the Levenberg-Marquardt algorithm with functions $A$ and $B$ respectively.
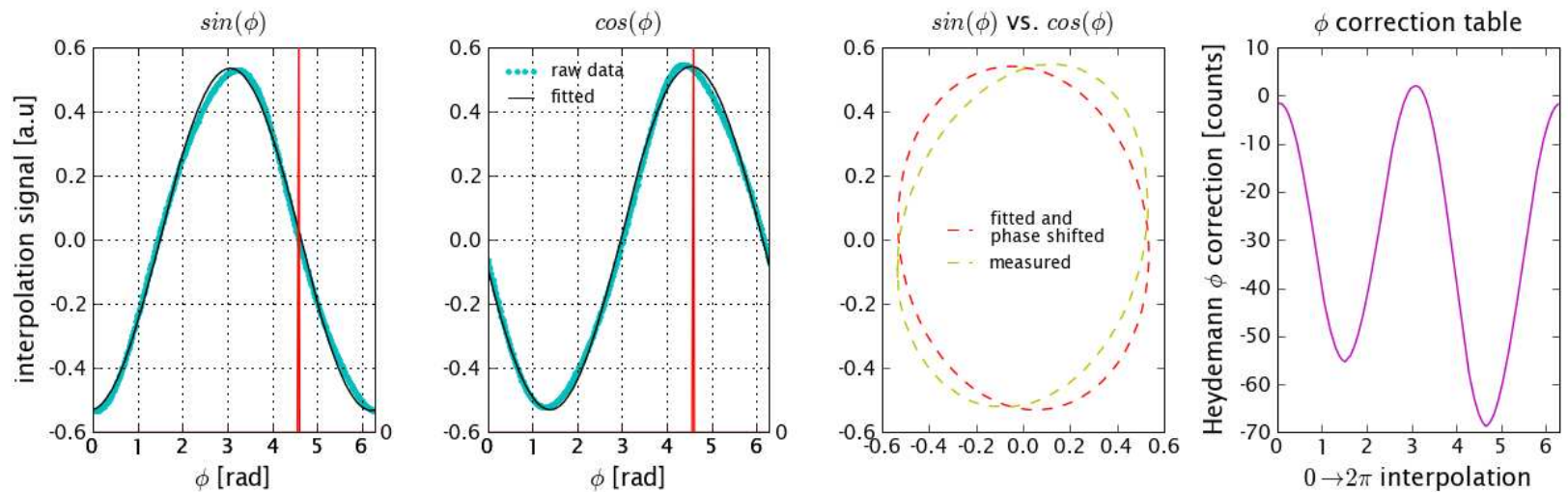

Figure 3. Heydemann correction for one encoder head (see text). Every encoder head has a unique correction table with two characteristic peaks.

The correction table is worked out by subtracting the ideal $\phi=a \tan (A / B)$ with no offsets, no phase shift and equal amplitudes, from the fitted $\operatorname{atan}(A / B)$ function. Since the correction table has to start at the beginning of the quadrature 
process, we set to $\phi_{A}=0$. Consequently we have to correct $\phi_{B} \rightarrow \phi_{B}-\phi_{A}$. The fitted and phase-shifted Lissajous oval is plotted in red-dashed line in Fig.3. Also for practical reasons the $\phi$-correction table with 0:2 $\pi$ interpolation interval is tabulated such that it corresponds to the length of the interpolation interval in raw encoder counts $(0: 2 \pi \rightarrow 0: 4096$ counts, because our RON 905 system is set such that the $0.01^{\circ}$ interpolation interval consists of 4096 encoder increments). The advantage is that in this way there is a fast and efficient software solution for the interpolation correction. Below is a function called corra () in Python that illustrates a simple computing machinery. It returns the corrected angle in degrees.

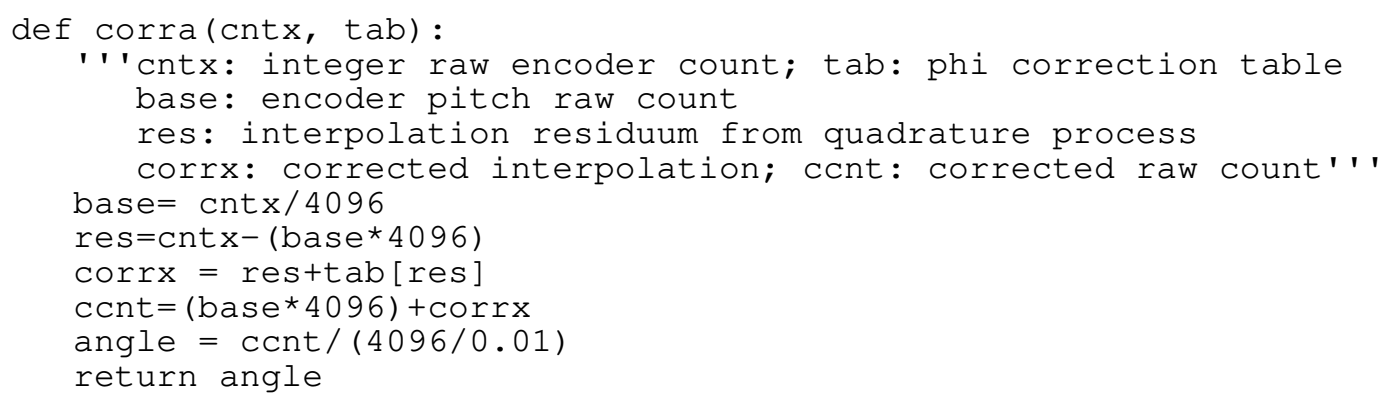

Finally let us note, that in Fig. 3 we did fit just one interpolation interval for the Heydemann correction. Since the $0.01^{\circ}$ interpolation interval was picked up randomly, the position of the encoder pitch, indicated in red line, is somewhere inside this span. In the above code snippet it is referred to the base variable. Note that the analog signals, as expected, are lined up with the encoder pitch positions. However, it turns out to be more adequate to fit more than one interpolation interval (say 5 to 10). The shape of the $\phi$-correction table does not change significantly but the amplitude may change.

Next section shows spectroscopic data that demonstrate the energy linearization with one single encoder head.

\section{EXPERIMENTAL RESULTS}

The need for the energy linearization in experiments becomes important in spectroscopy where the exact position of the peaks is required. This is also the case for example in high resolution RIXS spectroscopy. For example in the RIXS spectrometer SAXES [4] at the ADRESS beamline [5] the effects of the interpolation errors typically induce shifts in photon energy which impact on the resonance conditions and multiplet decays studied by RIXS. In order to verify the Heydemann correction, Fig.4 shows CO photoionization spectra from the ADRESS beamline which are entirely recorded within one grating interpolation interval (Fig.4a). We kept the mirror fixed at tree different positions within the $0.01^{\circ}$ mirror interpolation interval and we found accordingly the $0.01^{\circ}(36 \mathrm{arcsec})$ grating angular span that records the whole photoionization spectrum (Fig. 4 a-a'). Then we moved the mirror to a location shifted by $\sim 0.6^{\circ}$ and the whole procedure was repeated (Fig.4 b-b'; the PGM magnification ratio $c$ was changed from a value of $\sim 4$ to a value of $\sim 2$ ). In this way we assume that each spectrum is recorded at different locations of the grating and mirror interpolations interval. Spectra in Fig.4a-b are plotted against non-corrected energy readback, for the remaining spectra the energy readback was recalculated by means of Eq. 1 from the corrected mirror and grating angles $\theta$ and $\beta$ respectively. If the correction scheme is correct, the position of the CO-vibrational peaks should line-up for all spectra.

Fig.4a-b shows spectra without Heydemann correction; Fig.4 a'-b' zoom into the absorption fine-structure in order to demonstrate the effects when the correction is turned on and off. The effect is quite convincing: peak positions changing between 50-150 meV lineup such that the vibrational peaks are overlapping consistently. Their relative position significantly improves down to $5-10 \mathrm{meV}$ peak-to-peak. In order to demonstrate how all peaks line up with the correction algorithm, Fig.4c shows all spectra plotted relative to the maximum vibration peak (corrected spectra from Fig.4a' -b' were interpolated and smoothed). The peak positions nicely line up. We note that the spectral shape change for spectra recorded with different ratio $c$. This is not surprising: the PGM energy resolution change for different $c$.

Since the PGM mirror and grating absolute angles used for the energy readback were calibrated without the Heydemann correction, there is a small energy offset in the PGM energy readback between Fig.4a'-b'. The correction method has to be applied to each encoder head independently and the PGM needs to be recalibrated such that the averaged value from all encoders is used for the energy readback. 

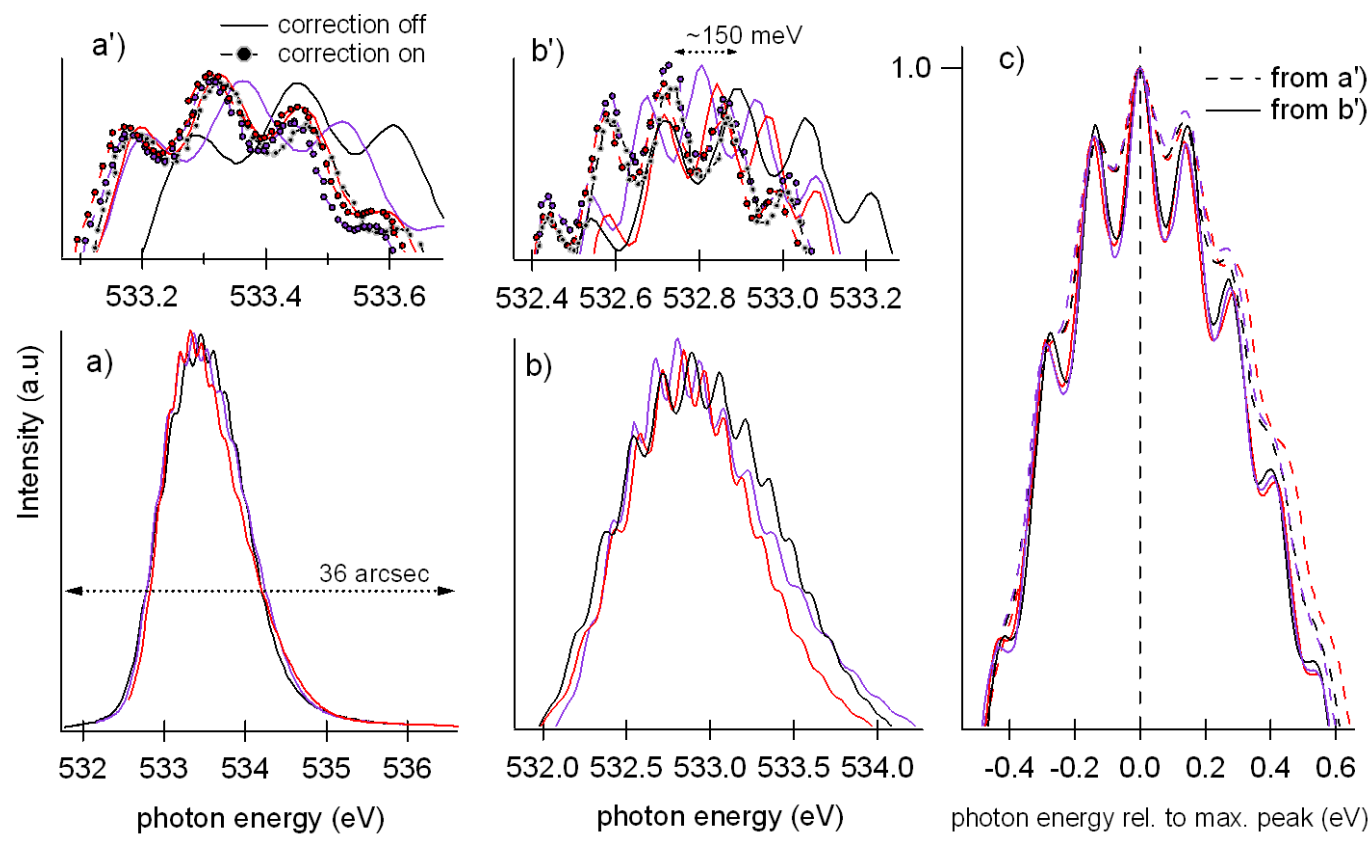

Figure 4. Verification of the Heydemann correction by means of CO-photoionization spectra. Spectra were measured by rotating the grating for tree different mirror positions. The whole spectrum fits into the encoder interpolation interval (36 arcsec). (a) and (b) show not corrected spectra for different magnification ratios $c$. (a') and (b') zoom into the vibrational structure in order to compare the spectra measured with and without the correction. (c) all spectra are aligned to the maximum peak. Data were recorded with 800 1/mm grating.

\section{SUMMARY}

The interpolation errors do not impact on the monochromator energy resolution (E/E $\approx \approx 10000$ for the ADRESS beamline), but randomly shift the energy on a scale larger than the spectroscopic resolution $(50-150 \mathrm{meV})$. We demonstrated the applicability of the Heydemann correction for improving the linearity of the monochromator energy. The method was verified with an autocollimator setup and by taking vibration spectra of $\mathrm{CO}$ gas. It shows that the Heydemann correction significantly improves the energy linearization on small energy scales of the order of $1 \mathrm{eV}$ down to 5-15 meV peak-to-peak.

\section{REFERENCES}

[1] P.L.M Heydemann, Applied Optics 20, 3382-3384 (1981).

[2] R.Follath, A. Balzer, SRI 2009 Proceeding

[3] www.heidenhain.de

[4] G. Ghiringhelli, A. Piazzalunga, C. Dallera, G. Trezzi, L. Braicovich, T. Schmitt, V.N. Strocov, R. Betemps, L. Patthey, X. Wang, M. Grioni, Rev. Sci. Instrum. 77 (2006) 113108.

[5] V.N. Strocov, T. Schmitt, U. Flechsig, T. Schmidt, A. Imhof, Q. Chen, J. Raabe, R. Betemps, D. Zimoch, J. Krempaský, X. Wang, M. Grioni, A. Piazzalunga, L.Patthey, J. Synchrotron Radiat. 17 (2010) 631-643. 